\title{
Nurse prescribing in primary care: a metasynthesis of the literature
}

\section{Dilyse Nuttall}

Principal Lecturer, School of Community Health and Midwifery, University of Central Lancashire, Preston, Lancsashire, UK

\begin{abstract}
Aim: The aim of this metasynthesis was to develop an understanding of the existing theoretical perspectives around nurse prescribing and to identify any gaps in knowledge, which would support further research into the lived experience of the nurse prescriber in the primary care setting. Background: Nurse prescribing has been the focus of many research studies since its introduction, with many benefits to the patient, the prescriber and service identified; however, there remains variation in the utilisation of the prescribing qualification, particularly in primary care settings. Although a range of quantitative and qualitative studies have been undertaken, which aimed to explore the influences on prescribing, few have used a research methodology that supports the in-depth exploration of the nurse prescriber's experience. Methods: An extensive literature search was undertaken in April 2015 (20-24), which included UK and non-UK studies since 1999. Inclusion and exclusion criteria were applied to search for studies in which participants included nurse prescribers who practiced in primary or community care settings. Studies that only used a quantitative methodology and those not available in English were excluded. The literature search yielded 124 papers, with 50 papers remaining after the initial screen of full papers against the inclusion/exclusion criteria. The papers were reviewed and graded for their quality, with a further 13 papers excluded.

A three-step qualitative analysis technique of metasynthesis was applied to the remaining 37 papers. Identification of similarities and differences enabled first-order interpretations to be identified, which were grouped into broader themes (second-order interpretations) by identifying concepts that applied to two or more studies. Further interpretation through synthesis of translation enabled third-order interpretations to emerge. Findings: From the metasynthesis of the 37 papers, nine themes emerged: patient-centred care; benefits to the service; the need for knowledge; professional accountability and boundary setting; safety consciousness; barriers to effective prescribing; role preservation; power-shifts and interprofessional relationships; and culture of prescribing.
\end{abstract}

Key words: metasynthesis; nurse prescribing; primary care

Received 7 February 2017; revised 5 July 2017; accepted 10 July 2017; first published online 8 August 2017

\section{Introduction}

Nurse prescribing is increasingly being recognised as an important activity in nursing practice, with countries including Spain, Norway, Finland,

Correspondence to: Dilyse Nuttall, Principal Lecturer, School of Community Health and Midwifery, University of Central Lancashire, Preston, Lancsashire PR1 2HE, UK. Email: dnuttall@uclan.ac.uk
Sweden, Ireland, The Netherlands, New Zealand, South Africa, Colombia, Australia, Canada and the United States of America enabling nurses to legally prescribe (Kroezen et al., 2012; Gielen et al., 2014; Weeks et al., 2016); however, the application of nurse prescribing varies between countries, with some, including the United Kingdom, enabling independent prescribing, and others applying strict conditions and supervision (Kroezen et al., 2012). In the United Kingdom, the Royal College of

(C) Cambridge University Press 2017 
Nursing (2012) identified that there were 54000 nurses and midwives qualified to prescribe in 2012. Of these, 35000 were Community Practitioner Nurse Prescribers (CPNPs), with the remaining 19000 being independent and supplementary prescribers. Figures presented in Health Education North West's (2015) large-scale economic evaluation of non-medical prescribing (NMP), suggested that although numbers in secondary care are increasing, there continues to be more (non-medical) prescribers in primary and community care settings. Despite the steady rise in nurse prescriber numbers, there is evidence that some nurse prescribers choose not to prescribe (Earle et al., 2011) whereas others do not prescribe to their full potential (Coull et al., 2013).

The primary focus of research in NMP to date has been on its impact, identifying an overall positive effect on patients, practitioners and the service (Courtenay et al., 2006; Bissell et al., 2008; Hacking and Taylor, 2010; Gielen et al., 2014; Health Education North West, 2015). In addition to the range of benefits, these and other studies identified a number of barriers to effective prescribing. Watterson et al.'s (2009) Scottish study found that prescribing was inhibited by a range of issues, which included a lack of access to training and a lack of support; however, the study also identified motivators, such as job satisfaction and the opportunity to improve patient care, which were linked to increased prescribing activity. It is evident that there are wide-ranging influences on nurses' prescribing, including the trust of other members of the team (Bowskill et al., 2013), the prescriber's confidence (Downer and Shepherd, 2010), their experience (Hall et al., 2008), the expectations of others (Nolan and Bradley, 2007) and the legislative controls enforced by different countries (Kroezen et al., 2012).

Although there have been numerous studies with a focus on nurse prescribing, there is still much more to discover. Many of the themes emerging from previous studies, such as confidence, competence and changes in role and relationships, suggest that the individual lived experiences of nurse prescribers are worthy of further exploration. There appears to be a need for a clearer insight into experiences and perceptions in prescribing practice in order to identify more effective ways to motivate and support nurse prescribers to prescribe more effectively. It is important to note that although many of the larger studies, such as those of Latter et al. (2012) and Health Education North West (2015) acknowledged that NMP in the United Kingdom was still predominantly undertaken in the primary care setting, many did not include CPNPs. As such, there is a clear need to learn from the lived experiences of our current prescribers in order to support them and our future NMPs more effectively.

This metasynthesis formed part of phase one of a phenomenological study aiming to explore the lived experience of the nurse prescriber in the community or primary care setting in the United Kingdom. The aim of the metasynthesis was to develop an understanding of the existing theoretical perspectives around nurse prescribing in order to identify any gaps in knowledge and any potential for new ideas.

\section{Method}

\section{Metasynthesis}

Systematic review utilises transparent, structured processes to review literature and this approach is equally important when reviewing qualitative literature as it requires the identification of clear criteria to support credibility, transferability, dependability and confirmability (Bearman and Dawson, 2013). Metasynthesis is a systematic approach to the synthesis of findings from qualitative studies, allowing key ideas and concepts to be identified (Erwin et al., 2011) while clearly identifying the quality measures employed throughout the process. This iterative approach enables a focus on the question being asked (Downe, 2008) and can introduce an additional layer of interpretation to those identified by the authors of the individual studies (Bearman and Dawson, 2013).

\section{Literature search}

The literature search was undertaken in April 2015 (20-24), using a range of databases and resources selected because of their relevance to the subject area (Table 1). In order to gather an insight into factors that could impact the lived experience of the nurse prescriber, a broad approach was applied to the literature search, 
Table 1 Databases and resources included in the literature search

\begin{tabular}{ll}
\hline Databases & Others \\
\hline CINAHL & Gov.UK \\
MEDLINE/OVID MEDLINE ACADEMIC SEARCH COMPLETE & $\begin{array}{l}\text { NICE Evidence Search Health \& } \\
\text { Social Care }\end{array}$ \\
PROQUEST HEALTH \& MEDICAL COMPLETE & Conference Papers/Presentations \\
OVID & \\
JOURNALS@ OVID & \\
EMBASE & \\
ERIC & \\
HMIC & \\
ELECTRONIC THESIS ONLINE SERVICE (EThOS) & \\
\hline
\end{tabular}

Table 2 Search strategy

\begin{tabular}{ll}
\hline Included criteria & Independent nurse prescribers practicing in primary/community care \\
& Supplementary nurse prescribers practicing in primary/community care \\
V100 prescribers & V150 prescribers \\
& V300 prescribers \\
Excluded criteria & Other prescribing groups: doctors, physicians, pharmacists, physiotherapists, \\
& radiographers, podiatrists/chiropodists, optometrists, dentists \\
& Patient perspectives \\
& Non-medical prescribing leads/managers \\
& Non-prescribing colleagues \\
& Nurse prescribers not practicing in primary/community care \\
& Studies not available in English \\
& Studies conducted pre-1999 \\
& Wholly quantitative methodologies \\
& Non-peer reviewed journals \\
Search terms & Nurse/Nurses/Nursing (Nurs*) \\
P (population) & Health Visitor/Health Visiting ('Health Visit*') \\
& District Nurse/District Nursing ('District Nurs*') \\
& Community Nurse/Community Nursing ('Community Nurs*') \\
& Primary Care ('Primary Care*) \\
I (issue) & Prescriber/Prescribers/Prescribing (Prescrib*) \\
E (Effect/method) & Qualitative \\
\hline
\end{tabular}

which included qualitative and mixed-methods papers. As such, the literature search included other reviews as well as primary studies. Studies that only gathered quantitative data were excluded as it was felt that the methodologies used would not provide data with the depth necessary to be relevant to a phenomenological study. International literature was included in the search, excluding only those not available in English. As the aim was to explore the perspectives and experiences of the nurse prescriber, studies that only explored the perspectives of other prescribers (doctors, physicians, pharmacists, physiotherapists, radiographers, podiatrists/chiropodists, optometrists and dentists), other staff or patients were excluded. Relevant professional nursing groups with prescribing rights were included (independent nurse prescribers; supplementary nurse prescribers; V100; V150; and V300) but this was limited to those practicing in a community or primary care setting. Although no lateral searches were undertaken, search terms were tested using Medical Subject Headings to ensure that terms used in the final searches captured all relevant data. Inclusion and exclusion criteria are identified in Table 2. The outcome of each stage of the literature search process is recorded in Figure 1.

Primary Health Care Research \& Development 2018; 19: 7-22 


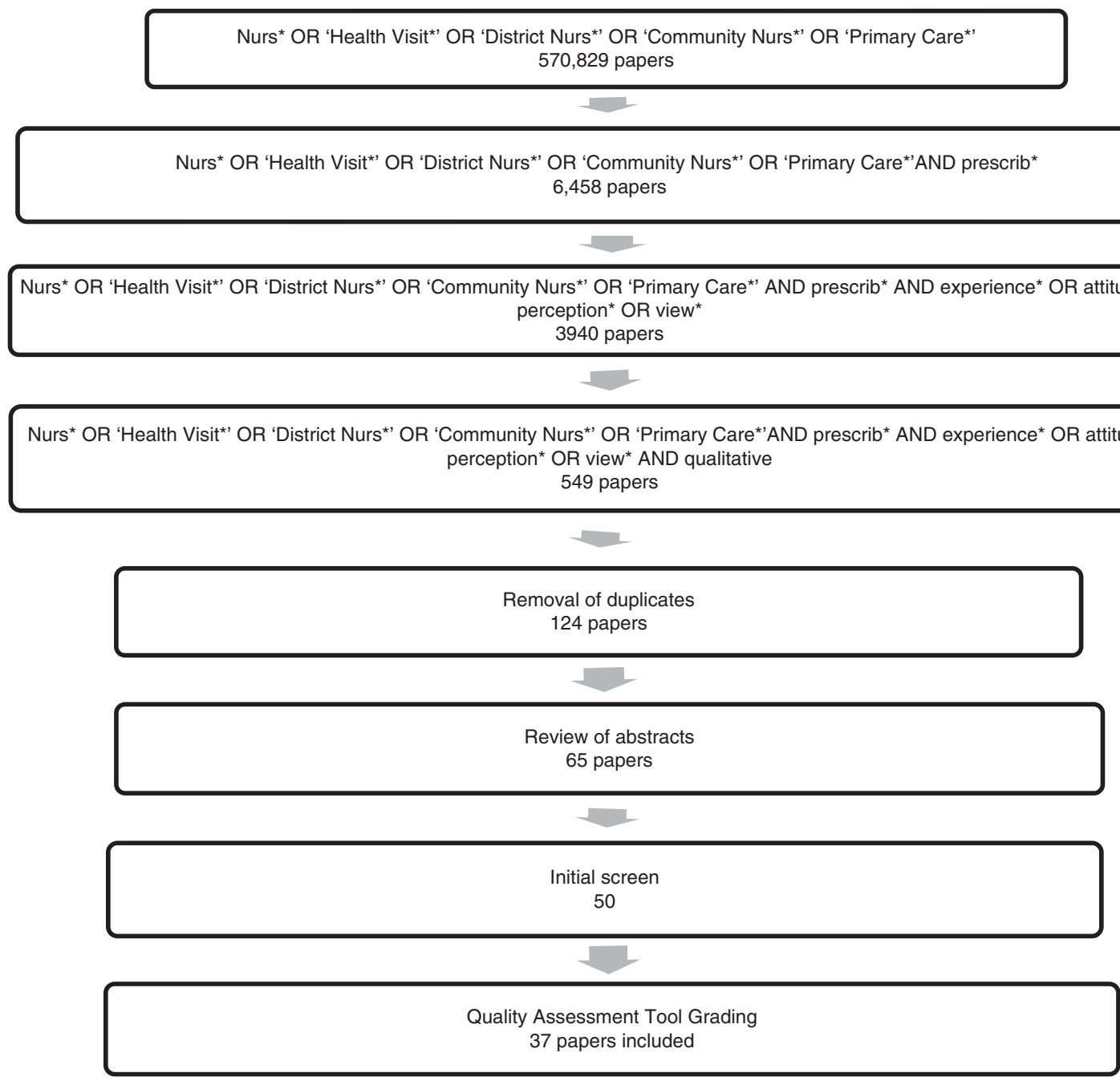

Figure 1 Literature search and quality assessment process.

\section{Qualitative analysis techniques}

Walsh and Downe's (2005) qualitative analysis technique was used, which 'attempts to integrate results from a number of different but inter-related qualitative studies' and, as such, was seen as appropriate for analysis of the range of data yielded from this literature search. In support of this technique, an adaptation of the Template for Metasynthesis and the grading system developed by Downe et al. (2009) were utilised. The first stage of Downe et al.'s (2009) approach required an initial screening of the full-text papers, resulting in 15 papers being rejected as the inclusion criteria Primary Health Care Research \& Development 2018; 19: 7-22 were not met, a factor not clear on initial reading of the abstracts. The quality of the remaining 50 papers was reviewed by the author and two other members of the research project supervisory team, using the Quality Assessment Tool questions shown in Table 3. This stage of the qualitative analysis process gave consideration to the clarity of the aims, appropriateness of the sample, design and methodology, as well as provided evidence that the findings were justified. The review also considered reflexivity, ethical issues and rigour. On the basis of the quality assessment, papers were graded using Downe et al.'s (2009) grading system 
Table 3 Quality assessment tool (Downe et al., 2009)

Are the aims clear?

Are the participants appropriate for the research question? Is the design appropriate for the aims and theoretical perspective?

Is the method(s) appropriate for the design?

Is the sample size and sampling justified?

Does the data analysis fit with the methodology?

Is reflexivity present?

Is the study ethical?

Does the data justify the findings?

Is the context described sufficiently?

Is there sufficient evidence of rigour?

Table 4 Grading system (Downe et al., 2009)

A: No, or few flaws. The study credibility, transferability, dependability and confirmability is high

B: Some flaws, unlikely to affect the credibility, transferability, dependability and/or confirmability of the study

C: Some flaws that may affect the credibility, transferability, dependability and/or confirmability of the study

D: Significant flaws that are very likely to affect the credibility, transferability, dependability and/or confirmability of the study

(Table 4) in which failure to meet any of the quality measures was identified as a flaw. The final grades were agreed upon by the three reviewers. In view of significant flaws evident in the papers graded D, these were excluded. Although acknowledging that the papers graded $\mathrm{C}$ contained some flaws that may affect credibility, transferability, dependability and/or confirmability, it was deemed that they added to the overall data and met the initial inclusion criteria. After exclusion of the 13 papers graded D, 37 remained and were included in the metasynthesis.

Participants in the included papers represented nurse prescribers from a range of countries: United Kingdom, United States of America, Canada, Botswana, Zimbabwe, Australia, Ireland, New Zealand and Sweden. Of the 37 papers, 23 were qualitative studies, whereas 11 used mixed methods. Qualitative data from four of the mixedmethods studies were collected using surveys or questionnaires that incorporated open questions, with the remainder also using either interviews or focus groups. Only the qualitative data from the mixed-methods papers were used for this metasynthesis; however, it is important to acknowledge that, in some of the studies, it was not always possible to clearly determine whether the themes identified by the authors were based solely on the qualitative data.

The final three papers were reviews of other studies (two literature reviews and one metasynthesis). Consistency of the findings of these three papers, with the data cited from the primary studies, was reviewed. The process used by the authors to assess the quality of the papers they included was also reviewed. The rigour of this process was used to inform the grade awarded to each of the three studies. Darvishpour et al.'s (2014) metasynthesis included 11 papers that met their inclusion criteria. The quality of the included papers was assessed using both the AMSTAR tool and the CASP tool, with all papers graded as either strong or moderate with both tools. Similarly, the systematic review by Bhanbhro et al. (2011) used the CASP tool, and of the 23 papers identified, six were excluded following quality review, because of insufficient detail on the research design and/or methodology used. Although the scores were not stated, the detail provided in the supplementary appraisal notes were suggestive of the quality of all included papers being strong or moderate. Harris and Taylor's (2004) literature review included 44 papers that met the inclusion criteria. The quality of these was assessed using an evaluation tool devised by the authors, which included review of the evidence typologies used. Again, the detail provided in the evaluation matrix aligned the quality of all included papers to grades of strong or moderate. The characteristics of the 37 studies are identified in Table 5.

Walsh and Downe (2006) identify a three-step approach to metasynthesis, which begins by identifying relationships or differences in the studies by comparing and contrasting the data. The next stage, reciprocal translation, attempts to translate the findings of one study into another by identifying concepts that could apply to both studies. Walsh and Downe's (2006) final stage is that of synthesis of translation, where the reciprocal translations are synthesised to identify new concepts or develop a more enhanced meaning. This approach was used by the author and nine themes were identified from the literature (Table 6). These themes were agreed upon by two other members of the supervisory team.

Primary Health Care Research \& Development 2018; 19: 7-22 
Table 5 Characteristics of papers included in metasynthesis

\begin{tabular}{|c|c|c|c|}
\hline Code & Author, date and country & Title & Design/method \\
\hline 1 & $\begin{array}{l}\text { Bhanbhro, S., Drennan, V.M., } \\
\text { Grant, R., \& Harris, R. (2011) } \\
\text { UK, USA, Canada, Botswana, } \\
\text { Zimbabwe }\end{array}$ & $\begin{array}{l}\text { Assessing the contribution of prescribing in } \\
\text { primary care by nurses \& professionals allied to } \\
\text { medicine: a systematic review }\end{array}$ & $\begin{array}{l}\text { Integrative review of } \\
\text { literature }\end{array}$ \\
\hline 2 & $\begin{array}{l}\text { Bowden, L. (2004) } \\
\text { England }\end{array}$ & $\begin{array}{l}\text { The impact of nurse prescribing on the role of the } \\
\text { district nurse }\end{array}$ & $\begin{array}{l}\text { Qualitative } \\
\text { interviews }\end{array}$ \\
\hline 3 & $\begin{array}{l}\text { Bowskill, D. (2009) } \\
\text { England }\end{array}$ & $\begin{array}{l}\text { The integration of nurse prescribing: case studies in } \\
\text { primary \& secondary care }\end{array}$ & $\begin{array}{l}\text { Qualitative } \\
\text { Case study design } \\
\text { Semi-structured interviews }\end{array}$ \\
\hline 4 & $\begin{array}{l}\text { Bradley, E., Hynam, B. \& } \\
\text { Nolan, P. (2007) } \\
\text { England }\end{array}$ & Nurse prescribing: reflections on safety in practice & $\begin{array}{l}\text { Qualitative } \\
\text { Interviews }\end{array}$ \\
\hline 5 & $\begin{array}{l}\text { Bradley, E. \& Nolan, P. (2007) } \\
\text { England }\end{array}$ & Impact of nurse prescribing: a qualitative study & $\begin{array}{l}\text { Qualitative } \\
\text { Grounded theory approach } \\
\text { Semi-structured interviews }\end{array}$ \\
\hline 6 & $\begin{array}{l}\text { Brodie, L., Donaldson, J. \& } \\
\text { Watt, S. (2014) } \\
\text { Scotland }\end{array}$ & $\begin{array}{l}\text { Non-medical prescribers \& benzo-diazepines: a } \\
\text { qualitative study }\end{array}$ & $\begin{array}{l}\text { Qualitative } \\
\text { Semi-structured interviews }\end{array}$ \\
\hline 7 & $\begin{array}{l}\text { Carey, N., Courtenay, M. \& } \\
\text { Burke, J. (2007) } \\
\text { UK-wide }\end{array}$ & $\begin{array}{l}\text { Supplementary nurse prescribing for patients with skin } \\
\text { conditions: a national questionnaire survey }\end{array}$ & $\begin{array}{l}\text { Mixed methods } \\
\text { Self-completed } \\
\text { questionnaire }\end{array}$ \\
\hline 8 & $\begin{array}{l}\text { Carey, N., Stenner, K. \& } \\
\text { Courtenay, M. (2014) } \\
\text { England }\end{array}$ & $\begin{array}{l}\text { An exploration of how nurse prescribing is being used } \\
\text { for patients with respiratory conditions across the east } \\
\text { of England }\end{array}$ & $\begin{array}{l}\text { Qualitative } \\
\text { Semi-structured telephone } \\
\text { interviews }\end{array}$ \\
\hline 9 & $\begin{array}{l}\text { Carey, N., Stenner, K. \& } \\
\text { Courtney, M. (2010) } \\
\text { England }\end{array}$ & $\begin{array}{l}\text { How nurse prescribing is being used in diabetes } \\
\text { services: views of nurses \& team members }\end{array}$ & $\begin{array}{l}\text { Qualitative } \\
\text { Semi-structured interviews }\end{array}$ \\
\hline 10 & $\begin{array}{l}\text { Coull, A., Murray, I., Turner-Halliday, F. } \\
\& \text { Watterson, A. (2013) } \\
\text { Scotland }\end{array}$ & $\begin{array}{l}\text { The expansion of nurse prescribing in Scotland: an } \\
\text { evaluation }\end{array}$ & $\begin{array}{l}\text { Mixed method } \\
\text { Omnibus survey } \\
\text { Case study interviews }\end{array}$ \\
\hline 11 & $\begin{array}{l}\text { Cousins, R. \& Donnell, C. (2012) } \\
\text { England }\end{array}$ & $\begin{array}{l}\text { Nurse prescribing in general practice: a qualitative study } \\
\text { of job satisfaction \& work-related stress }\end{array}$ & $\begin{array}{l}\text { Qualitative } \\
\text { In-depth interviews }\end{array}$ \\
\hline 12 & $\begin{array}{l}\text { Darvishpour, A, Joolaee, S. \& } \\
\text { Cheraghi, M.A. (2014) } \\
\text { UK, USA, Canada, Botswana, } \\
\text { Zimbabwe, Australia, Ireland, } \\
\text { New Zealand, Sweden }\end{array}$ & $\begin{array}{l}\text { A meta-synthesis study of literature review \& systematic } \\
\text { review published in nurse prescribing }\end{array}$ & Qualitative meta-synthesis \\
\hline 13 & $\begin{array}{l}\text { Davies, J. (2005) } \\
\text { England }\end{array}$ & $\begin{array}{l}\text { Health visitors' perceptions of nurse prescribing: a } \\
\text { qualitative field work study }\end{array}$ & $\begin{array}{l}\text { Qualitative } \\
\text { Interviews }\end{array}$ \\
\hline 14 & $\begin{array}{l}\text { DH (2011) } \\
\text { UK }\end{array}$ & $\begin{array}{l}\text { Evaluation of nurse \& pharmacist independent } \\
\text { prescribing }\end{array}$ & $\begin{array}{l}\text { Mixed methods } \\
\text { Questionnaire survey, focus } \\
\text { groups, interviews \& } \\
\text { workshops }\end{array}$ \\
\hline 15 & $\begin{array}{l}\text { Downer, F. \& Shepherd, C.M. (2010) } \\
\text { Scotland }\end{array}$ & $\begin{array}{l}\text { District nurses prescribing as nurse independent } \\
\text { prescribers }\end{array}$ & $\begin{array}{l}\text { Qualitative } \\
\text { Heideggerian } \\
\text { phenomenology } \\
\text { Interviews }\end{array}$ \\
\hline 16 & $\begin{array}{l}\text { Fisher, R. (2010) } \\
\text { England }\end{array}$ & $\begin{array}{l}\text { Nurse prescribing: a vehicle for improved collaboration, } \\
\text { or a stumbling block to inter-professional working? }\end{array}$ & $\begin{array}{l}\text { Qualitative } \\
\text { Ethnographic } \\
\text { Interviews and observations }\end{array}$ \\
\hline 17 & $\begin{array}{l}\text { Hall, J. (2006) } \\
\text { UK }\end{array}$ & Influences on community nurse prescribing & $\begin{array}{l}\text { Mixed methods } \\
\text { Semi-structured interviews } \\
\text { Questionnaire }\end{array}$ \\
\hline 18 & $\begin{array}{l}\text { Hall, J., Cantrill, J. \& Noyce, P. (2006) } \\
\text { England }\end{array}$ & $\begin{array}{l}\text { Why don't trained community nurse prescribers } \\
\text { prescribe? }\end{array}$ & $\begin{array}{l}\text { Mixed methods } \\
\text { Semi- structured interviews } \\
\text { Questionnaire }\end{array}$ \\
\hline 19 & $\begin{array}{l}\text { Hall, J., Cantrill, J. \& Noyce, P. (2004) } \\
\text { England }\end{array}$ & $\begin{array}{l}\text { Managing independent prescribing: the influence of } \\
\text { primary care trusts on community nurse prescribing }\end{array}$ & $\begin{array}{l}\text { Qualitative } \\
\text { Semi-structured interviews }\end{array}$ \\
\hline 20 & $\begin{array}{l}\text { Hall, J., Cantrill, J. \& Noyce, P. (2003) } \\
\text { England }\end{array}$ & Influences on community nurse prescribing & $\begin{array}{l}\text { Qualitative } \\
\text { Semi-structured interviews }\end{array}$ \\
\hline 21 & $\begin{array}{l}\text { Harris, J. \& Taylor, J. (2004) } \\
\text { UK, Sweden, Australia, New Zealand, } \\
\text { USA }\end{array}$ & Research literature review on prescribing & $\begin{array}{l}\text { Literature review } \\
\text { Quantitative and qualitative } \\
\text { papers included }\end{array}$ \\
\hline
\end{tabular}


Table 5 (Continued)

\begin{tabular}{|c|c|c|c|}
\hline Code & Author, date and country & Title & Design/method \\
\hline 22 & $\begin{array}{l}\text { Jones, M., Bennett, J., Lucas, B., } \\
\text { Miller, D. \& Gray, R. (2007) } \\
\text { England }\end{array}$ & $\begin{array}{l}\text { Mental health nurse supplementary prescribing: } \\
\text { experiences of mental health nurses, psychiatrists and } \\
\text { patients }\end{array}$ & $\begin{array}{l}\text { Qualitative } \\
\text { Semi-structured interviews }\end{array}$ \\
\hline 23 & $\begin{array}{l}\text { Klein, T. (2015) } \\
\text { Oregon, USA }\end{array}$ & $\begin{array}{l}\text { Clinical nurse specialist prescriber characteristics \& } \\
\text { challenges in Oregon }\end{array}$ & $\begin{array}{l}\text { Mixed methods } \\
\text { Descriptive survey }\end{array}$ \\
\hline 24 & $\begin{array}{l}\text { Lewis-Evans, A. \& Jester, R. (2004) } \\
\text { England }\end{array}$ & Nurse prescribers' experiences of prescribing & $\begin{array}{l}\text { Qualitative } \\
\text { Minimally structured } \\
\text { interviews }\end{array}$ \\
\hline 25 & $\begin{array}{l}\text { Luker, K.A. \& McHugh, G.A. (2002) } \\
\text { UK }\end{array}$ & $\begin{array}{l}\text { Nurse prescribing from the community nurse's } \\
\text { perspective }\end{array}$ & $\begin{array}{l}\text { Mixed methods } \\
\text { Postal questionnaire } \\
\text { Mainly open-ended } \\
\text { questions }\end{array}$ \\
\hline 26 & $\begin{array}{l}\text { Maddox, C. (2011) } \\
\text { England }\end{array}$ & $\begin{array}{l}\text { Influences on non-medical prescribing: nurse \& } \\
\text { pharmacist prescribers in primary \& community care }\end{array}$ & $\begin{array}{l}\text { Qualitative } \\
\text { In-depth interviews } \\
\text { Critical incident technique }\end{array}$ \\
\hline 27 & $\begin{array}{l}\text { Mahoney, D.F. \& Ladd, E. (2010) } \\
\text { USA }\end{array}$ & $\begin{array}{l}\text { More than a prescriber: gerontological nurse } \\
\text { practitioners' perspectives on prescribing \& } \\
\text { pharmaceutical marketing }\end{array}$ & $\begin{array}{l}\text { Qualitative } \\
\text { Focus groups }\end{array}$ \\
\hline 28 & $\begin{array}{l}\text { NIPEC (2007) } \\
\text { Northern Ireland }\end{array}$ & $\begin{array}{l}\text { Review of the implementation of the nurse prescriber } \\
\text { role }\end{array}$ & $\begin{array}{l}\text { Mixed methods } \\
\text { Questionnaire } \\
\text { Focus groups workshops }\end{array}$ \\
\hline 29 & $\begin{array}{l}\text { Ross, J.D., Clarke, A. \& Kettles, A.M. } \\
(2014) \\
\text { England }\end{array}$ & $\begin{array}{l}\text { Mental health nurse prescribing: using a constructivist } \\
\text { approach to investigate the nurse-patient relationship }\end{array}$ & $\begin{array}{l}\text { Qualitative } \\
\text { constructivist } \\
\text { Focus groups interviews }\end{array}$ \\
\hline 30 & $\begin{array}{l}\text { Scottish Government (2009) } \\
\text { Scotland }\end{array}$ & An evaluation of nurse prescribing in Scotland & $\begin{array}{l}\text { Mixed methods } \\
\text { Questionnaire } \\
\text { Survey } \\
\text { Case studies } \\
\text { Interviews }\end{array}$ \\
\hline 31 & $\begin{array}{l}\text { Sodha, M., McLaughlin, M., } \\
\text { Williams, G. \& Dhillon, S. (2002) } \\
\text { England }\end{array}$ & Nurses' confidence \& pharmaco-logical knowledge & $\begin{array}{l}\text { Mixed methods } \\
\text { Cross-sectional survey } \\
\text { Open and closed questions }\end{array}$ \\
\hline 32 & $\begin{array}{l}\text { Spitz, A., Moore, A., A., Papaleontio, M., } \\
\text { Granieri, E., Turner, B., J., \& Reid, M. } \\
\text { (2011) } \\
\text { New York, USA }\end{array}$ & $\begin{array}{l}\text { Primary care providers' perspective on prescribing } \\
\text { opioids to older adults with chronic non-cancer pain: } \\
\text { a qualitative study }\end{array}$ & $\begin{array}{l}\text { Qualitative } \\
\text { Cross-sectional study } \\
\text { Focus groups }\end{array}$ \\
\hline 33 & $\begin{array}{l}\text { Stenner, K., Carey, N., \& Courtenay, M. } \\
(2010) \\
\text { England }\end{array}$ & How nurse prescribing influences the role of nursing & $\begin{array}{l}\text { Qualitative } \\
\text { Case study approach } \\
\text { Interviews }\end{array}$ \\
\hline 34 & $\begin{array}{l}\text { Stenner, K., Carey, N., \& Courtenay, M. } \\
(2010) \\
\text { England }\end{array}$ & $\begin{array}{l}\text { Implementing nurse prescribing: a case study in } \\
\text { diabetes }\end{array}$ & $\begin{array}{l}\text { Qualitative } \\
\text { Semi-structured interviews }\end{array}$ \\
\hline 35 & $\begin{array}{l}\text { Stenner, K., \& Courtenay, M. (2008) } \\
\text { England }\end{array}$ & $\begin{array}{l}\text { Benefits of nurse prescribing for patients in pain: nurses' } \\
\text { views }\end{array}$ & $\begin{array}{l}\text { Qualitative } \\
\text { Semi-structured interviews }\end{array}$ \\
\hline 36 & $\begin{array}{l}\text { Stenner, K., \& Courtenay, M. (2008) } \\
\text { England }\end{array}$ & $\begin{array}{l}\text { The role of inter-professional relationships \& support for } \\
\text { nurse prescribing in acute } \& \text { chronic pain }\end{array}$ & $\begin{array}{l}\text { Qualitative } \\
\text { Semi-structured interviews }\end{array}$ \\
\hline 37 & $\begin{array}{l}\text { Wilhelmsson S., \& Foldevi, M. (2003) } \\
\text { Sweden }\end{array}$ & $\begin{array}{l}\text { Exploring views on Swedish district nurses' prescribing: } \\
\text { a focus group study in primary care }\end{array}$ & $\begin{array}{l}\text { Qualitative } \\
\text { Focus group interviews }\end{array}$ \\
\hline
\end{tabular}

\section{Emerging themes}

\section{The need to provide patient-centred care}

The theme of 'the need to provide patientcentred care' was identified from 15 papers, two of which were literature reviews, one was a metasynthesis and three were mixed-methods studies. A benefit of nurse prescribing, which featured in many of the studies was the nurse prescriber's ability to provide and improve patient-centred care, suggesting a need to integrate care into prescribing by using a holistic approach. The district nurse prescribers interviewed in Bowden's (2005) and Downer and Shepherd's (2010) studies, referred to an improved or better quality of care as a positive outcome of NMP. Support for these findings was demonstrated in studies by Mahoney and Ladd (2010) and Carey et al. (2014) as well as by a metasynthesis by Darvishpour et al. (2014). A variety of factors influenced the nurse prescriber's Primary Health Care Research \& Development 2018; 19: 7-22 


\section{Dilyse Nuttall}

Table 6 Three-step approach to metasynthesis

\begin{tabular}{|c|c|c|c|}
\hline First-order interpretations & $\begin{array}{l}\text { Second-order } \\
\text { interpretations }\end{array}$ & Papers & $\begin{array}{l}\text { Third-order } \\
\text { interpretations }\end{array}$ \\
\hline $\begin{array}{l}\text { Efficiency, timeliness, waiting times, access to medicines, } \\
\text { reduced hospital admissions, use of skills, confidence in } \\
\text { NMP, more time with patient, acceptability, patient } \\
\text { satisfaction, ability to provide information, patient choice, } \\
\text { improved adherence, flexibility in appointment times, } \\
\text { concordance, minimal disruption, lack of established } \\
\text { relationship made nurses uneasy about prescribing, } \\
\text { patients expected more than nurses could offer }\end{array}$ & Patient impact & $\begin{array}{l}1,5,8,10,13,14,15,17,20 \\
21,24,27,35\end{array}$ & $\begin{array}{l}\text { The need to provide } \\
\text { patient-centred care }\end{array}$ \\
\hline $\begin{array}{l}\text { Seamless care, improved patient care, better patient care, } \\
\text { complete episodes of care, patient-centred, continuity } \\
\text { of care }\end{array}$ & Completing care & $\begin{array}{l}1,2,5,8,10,12,15,24 \\
30,35\end{array}$ & \\
\hline $\begin{array}{l}\text { Effectiveness of treatment, cost-effectiveness, resources, } \\
\text { nurses felt they were more aware of budgetary issues, } \\
\text { whereas doctors worried they would not be aware. External } \\
\text { pressures from their managers and formularies to } \\
\text { prescribe low-cost products, more time for consultations, } \\
\text { value to the service }\end{array}$ & Service impact & $1,2,10,13,17,20,24$ & $\begin{array}{l}\text { The benefits to the } \\
\text { service }\end{array}$ \\
\hline $\begin{array}{l}\text { Training, preparation for practice, assimilation of } \\
\text { knowledge, correlation between specialist training and } \\
\text { higher rates of prescribing, knowledgeable about } \\
\text { pharmacology, more knowledgeable, CPD, responsibility } \\
\text { to keep up to date }\end{array}$ & Knowledge & $\begin{array}{l}2,4,6,7,9,10,11,12,14 \\
15,17,19,21,22,25,30 \\
31,35,37\end{array}$ & $\begin{array}{l}\text { The need for } \\
\text { knowledge }\end{array}$ \\
\hline $\begin{array}{l}\text { Accountability, use of guidelines, those who did deviate } \\
\text { tended to be more experienced NPs. Views on formularies } \\
\text { were neutral (a guide) to negative (restrictive) }\end{array}$ & Accountability & $2,3,17,33$ & $\begin{array}{l}\text { Professional } \\
\text { accountability and } \\
\text { boundary setting }\end{array}$ \\
\hline $\begin{array}{l}\text { Confidence, comfort, familiar products, competence to } \\
\text { diagnose, decision-making, risk of becoming over } \\
\text { confident, over-estimation of competence, increased } \\
\text { anxiety with increased responsibility, less comfortable } \\
\text { prescribing items for first time or that they rarely } \\
\text { prescribed, more comfortable prescribing items associated } \\
\text { with low risk), some products risky (paracetamol and } \\
\text { laxatives), less comfortable prescribing for the first time or } \\
\text { an item rarely prescribed }\end{array}$ & Competence & $\begin{array}{l}2,3,4,8,10,12,15,17,20 \\
25,26,31,35\end{array}$ & \\
\hline $\begin{array}{l}\text { Risk taking, safe and unsafe items, safer than by proxy, more } \\
\text { careful, audit, co-morbidities, concerns re-lack of } \\
\text { diagnostic expertise, cautious approach supported patient } \\
\text { safety, range of quality assurance tools and CPD activities } \\
\text { used, lack of experience in prescribing for particular age } \\
\text { groups, lack of access to records to determine underlying } \\
\text { conditions, allergies, if treatment had been prescribed } \\
\text { previously, more comfortable prescribing items pts have } \\
\text { had before, uncomfortable prescribing for a pt they did not } \\
\text { know, fear of making mistakes, reliability of patient and } \\
\text { caregiver }\end{array}$ & Avoiding harm & $\begin{array}{l}2,3,4,8,10,12,14,17,20 \\
22,26,30,32,35\end{array}$ & Safety consciousness \\
\hline $\begin{array}{l}\text { Chore of repeat prescribing, pressure to prescribe, } \\
\text { differences in practice, time, lack of support and CPD, cross- }\end{array}$ & Barriers & $\begin{array}{l}2,3,7,8,10,11,12,13,15 \\
17,18,26\end{array}$ & $\begin{array}{l}\text { Barriers to effective } \\
\text { prescribing }\end{array}$ \\
\hline
\end{tabular}
lack of reward, legal limitations, executive factors, educational deficiencies, research weaknesses, concerns re-pharmacological knowledge, lack of understanding of role, limited formulary, access to records, views on formularies ranged from neutral to negative, with them acting as a guide which was not required or restricting prescriber choice/professional freedom

Job satisfaction, improved professional role, being left

behind, self-empowerment, professionalism, working outside traditional boundaries, complement not replace doctors, new boundaries, traditional hierarchies, doctorchecking, exclusive to doctors, self-esteem, ability to challenge, legitimising nursing role, integrating caring and curing, medicalisation, add-on role, brand of prescriber, autonomy, status, respect from colleagues and patients, essential to specialist roles, shared territory, reaching full 
Table 6 (Continued)

\begin{tabular}{|c|c|c|c|}
\hline First-order interpretations & $\begin{array}{l}\text { Second-order } \\
\text { interpretations }\end{array}$ & Papers & $\begin{array}{l}\text { Third-order } \\
\text { interpretations }\end{array}$ \\
\hline \multicolumn{4}{|l|}{$\begin{array}{l}\text { potential, increased the respect they received from doctors, } \\
\text { used prescribing to complement nursing actions rather } \\
\text { than substitute other aspects of their role, job descriptions } \\
\text { should support the NP role }\end{array}$} \\
\hline $\begin{array}{l}\text { Improved relationship with pharmacist, collaborative } \\
\text { working, improved communication with colleagues, } \\
\text { doctors' time was also used more effectively to deal with } \\
\text { more complex cases, some doctors unclear about nurses } \\
\text { prescribing authority, good interdisciplinary } \\
\text { communication re prescribing in their area of practice, GPs } \\
\text { dictating what can be prescribed, access to GPs, control } \\
\text { and domination, inappropriate expectations, lack of } \\
\text { understanding of role, fear of exploitation, resistance from } \\
\text { colleagues, professional rivalry, no change in status, } \\
\text { change in content of conversations and in team } \\
\text { interactions, lack of reward }\end{array}$ & $\begin{array}{l}\text { Collaboration and } \\
\text { relationships with } \\
\text { colleagues }\end{array}$ & $\begin{array}{l}2,3,4,9,10,12,14,16,17 \\
24,33,34,36\end{array}$ & $\begin{array}{l}\text { Power-shifts in inter- } \\
\text { professional } \\
\text { relationships }\end{array}$ \\
\hline $\begin{array}{l}\text { Support, trust, link between support of doctor and } \\
\text { effectiveness }\end{array}$ & $\begin{array}{l}\text { Doctor's influence } \\
\text { and support }\end{array}$ & $\begin{array}{l}3,4,5,8,9,10,25,28,34 \\
36,37\end{array}$ & \\
\hline $\begin{array}{l}\text { Need for organisational support, fragmented } \\
\text { implementation of policy, NP was largely driven by the } \\
\text { practitioner to enhance existing services rather than enable } \\
\text { service re-design, only half of Trusts had a strategy for the } \\
\text { development of NMP, organisational preparedness }\end{array}$ & $\begin{array}{l}\text { Organisational } \\
\text { support }\end{array}$ & $1,3,5,10,14,22,34,36,37$ & Culture of prescribing \\
\hline
\end{tabular}

$\mathrm{NMP}=$ non-medical prescribing; $\mathrm{CPD}=$ continuing professional development; $\mathrm{NP}=$ nurse prescribers

ability to provide patient-centred care through prescribing, including the ability to increase effectiveness through the timely delivery of complete episodes of care (Bhanbhro et al., 2011). The importance of timeliness was reflected by LewisEvans and Jester (2004) and by Coull et al. (2013) in their Scottish study, specifically referring to NMPs having time to explain treatments. In addition, the ability to tailor prescribing to the needs of the patient was identified as a key enabler in studies by Hall (2006) and Stenner and Courtenay (2008b), with the desire to benefit the patient and to avoid harm identified as important.

The drive to provide patient-centred care through nurse prescribing activity, however, may not be without its consequences. Luker and McHugh (2002), who carried out a postal survey across three UK Primary Care Trusts, found that nurse prescribers sometimes felt that patients expected more from them than they were able to offer. Similarly, the 2011 large-scale study by the Department of Health (DH), identified that NMP does not enable all nurses to meet the needs of all patients, largely because of concerns about prescribing for patients with co-morbidities; however, it would be fair to suggest that this issue is not exclusive to prescribing.

\section{Benefits to the service}

In addition to benefiting the patient, seven of the papers, including one literature review and two mixed-methods studies, indicated that prescribing has a positive impact on the service in which it is implemented. Bhanbhro et al. (2011) identified a perception by nurses that their prescribing activity reduced doctors' workloads but as a consequence, nurses' workloads increased. Interestingly, doctors themselves did not recognise any reduction in their workload. It is worthy to note that nurse prescribers acknowledge time saved for other colleagues as a benefit, suggesting a shared responsibility for patient care and the ability (and willingness) to see benefits from a wider perspective. Davies (2005) also identified that prescribing could save time for doctors and for the patient themselves, but that this required them to spend more time with the patient. As a result, the extra time spent with one patient was often at the expense of the time available to other patients; however, some of the nurse prescribers in the study by Hall (2006) offered a degree of counterbalance, suggesting that the extra time that the nurse prescriber had to spend with individual patients (compared with GPs) meant they were able to undertake a more thorough assessment, a finding shared by Lewis-Evans and Jester (2004). As a 
consequence, they were able to fully explore the appropriate strategy and, therefore, were actually less likely to prescribe, supporting Coull et al.'s (2013) finding that NMP was perceived to result in a better use of resources and improved costeffectiveness. Importantly, the improved access and appropriate prescribing of medicines through NMP is seen as having the potential to manage the many demands on health services and, consequently, has the potential to reduce patient dissatisfaction (Bhanbhro et al., 2011).

\section{The need for knowledge}

An issue featuring in 19 of the papers, including seven mixed-methods papers, a metasynthesis and a literature review, was the need for adequate knowledge by nurse prescribers. This included the need for knowledge required to initiate prescribing following a programme of education and for on-going updates and continuing professional development (CPD) opportunities (Bowden, 2005). The Scottish Government (2009) found that nurses who had undertaken a prescribing programme generally felt that it was fit for purpose, equipping them to make more appropriate use of their skills and to be more effective as nurses (Coull et al., 2013); however, Hall (2006) identified the desire for on-going education amongst nurse prescribers and, additionally, the desire for feedback on their prescribing practice to ensure that this remained effective. The on-going nature of learning and development is supported by Coull et al. (2013) who found that the prescribing skills of the participants in their study appeared to have increased over time. Bowskill (2009) referred to this as 'an assimilation of knowledge' whereby nurse prescribers continue to gather knowledge as they prescribe, a concept also identified by Stenner and Courtenay (2008b).

Unfortunately, despite this need for knowledge and CPD, nurses prescribers had experienced difficulties in accessing it, particularly CPD arranged by the employers (Luker and McHugh, 2002; Harris and Taylor, 2004). Although acknowledging that these studies were undertaken relatively early on in the lifespan of nurse prescribing, the later study by Hall (2006) also found this to be the case. More recently, Cousins and Donnell's (2012) study, which focussed on job satisfaction and work-related stress in general practice, again identified a lack of access to updates and a lack of support from general practice for their CPD; however, it is noteworthy that nurses had differing perceptions of whose responsibility it was to provide CPD opportunities, with some accepting it as their own and actively seeking CPD activities, whereas others expected their employer to provide this for them (Hall, 2006). As such, the CPD accessed varied, with some seeking support from colleagues and using information from drug companies and journals to update themselves, whereas those in more specialised fields of practice accessed CPD that was more specialised (Luker and McHugh, 2002; Carey et al., 2007; Carey et al., 2010). The reality of the situation was effectively summarised by the Scottish Government (2009) who suggested that a lack of support for CPD could compromise patient safety.

\section{Professional accountability and boundary setting'}

In 10 of the qualitative papers, four of the mixed-methods papers and in the metasynthesis paper, the theme of 'professional accountability and boundary setting' emerged. Nurse prescribers demonstrated accountability in a range of guises, including awareness of boundaries, competence and confidence. The perception of accountability differed depending on the prescribing situation, particularly when prescribing products (or for patients), where the risk was perceived as greater (Bowden, 2005). A prominent factor linked to accountability was the use of formularies and guidelines in setting boundaries and supporting the nurse's prescribing practice. Stenner et al. (2010a; 2010b) found that nurse prescribers were more likely than GPs to follow guidelines and used these to support their decision-making, whereas deviation from these was linked to a higher level of decision-making, acknowledging the individual's accountability for complex decisions. Similarly, Hall (2006) found that nurse prescribers who did deviate from guidelines and formularies tended to be more experienced nurse prescribers.

Darvishpour et al. (2014) identified competence as one of the key facilitators of prescribing and having clear links to professional accountability and to the need for knowledge. Maddox (2011) aimed to examine factors that influenced competency in NMPs and found that even though they perceived themselves as competent, they were less 
sure that other health professionals were able to make that same judgement about them. The nurses in the study related their competence to their level of pharmaceutical knowledge, their skill in considering differential diagnoses and establishing a diagnosis and an ability to effectively monitor and modify the treatments prescribed.

Many studies that explored nurse prescribers' experiences identified confidence as a key influence on their prescribing activity. Stenner and Courtenay (2008b) reported an increased confidence in recommending medications amongst nurse prescribers, with Carey et al. (2014) similarly reporting a general increase in confidence. Interestingly, confidence was seen as developmental (Luker and McHugh, 2002; Downer and Shepherd, 2010), in that there was a perception of building up confidence to prescribe. In fact, lack of opportunity to build up and develop confidence was seen as a barrier to prescribing (Hall et al., 2003; Bradley et al., 2007). Completion of the prescribing programme was seen by nurse prescribers as a starting point from which confidence would grow, with many using their colleagues as a conduit for developing competence (Bradley et al., 2007). It is apparent that nurse prescribers give much consideration to their professional accountability and that they set boundaries on their prescribing practice in line with this.

\section{Safety consciousness}

Safety featured in four of the mixed-methods studies, the metasynthesis paper and in nine of the qualitative studies reviewed, with evidence of nurses adopting their prescribing practice to maintain the safety of their patients. Bradley et al. (2007) found that nurse prescribers perceived their current prescribing practice as safer than the 'by proxy prescribing' practice previously used, as they were prescribing on the basis of their own assessment of the patient's needs. Nurses were able to identify key processes and practices in prescribing as supporting safety, including accountability, accepting responsibility for keeping up to date and undertaking regular audits of their practice (Bradley et al., 2007); however, Carey et al. (2014) identified that the increased responsibility for nurse prescribers often resulted in increased anxiety, with nurses worrying about making mistakes or being penalised for their prescribing decisions. Interestingly, although there was no evidence of any mistakes having been made, the nurse prescribers in Jones et al.'s (2007) study identified concern about making mistakes as a barrier to prescribing, suggesting that nurse prescribers perceive a blame-culture.

Factors that nurse prescribers considered as risks emerged in a number of studies and were closely linked to the desire to avoid harm. Maddox (2011) identified that nurse prescribers perceived a 'degree of risk' with certain prescribing situations, such as prescribing outside of guidelines, prescribing high doses or off-label and prescribing high-risk medicines. Other concerns related specifically to characteristics of the patient, with some nurses feeling uncomfortable if prescribing for the very old and/or the very young (Hall et al., 2003). Both Maddox (2011) and Carey et al. (2014) identified that nurse prescribers appeared more concerned about safety when the patient had co-morbidities and were therefore considered to be at a higher risk, compounded in situations in which support for the nurse prescriber was not readily available. It is evident from the literature that a range of strategies are used by nurse prescribers to maintain the safety of their patients. A personal formulary was often used to support safe prescribing as it was exclusively made up of drugs that the nurse felt she/he was competent and safe to prescribe but with the decision made not to prescribe when there were safety concerns (Maddox, 2011). It would seem that nurse prescribers have a safety consciousness that influences their prescribing practice, although it is unclear whether this is as a result of prescribing or whether it is already inherent in their nursing practice.

\section{Barriers to effective prescribing}

Unsurprisingly, factors identified as supporting prescribing, featured as barriers when absent, a theme that emerged in the metasynthesis paper as well as in four of the mixed-methods studies and seven qualitative studies. A lack of pharmaceutical knowledge was acknowledged as a self-imposed factor in preventing nurse prescribers writing a prescription (Carey et al., 2007; Coull et al., 2013; Darvishpour et al., 2014). As such, when training and support were perceived to be lacking or inadequate, this was seen as a further barrier (Davies, 2005; Carey et al., 2014; Carey et al., 2007; Maddox, 2011; Cousins and Donnell, 2012;

Primary Health Care Research \& Development 2018; 19: 7-22 
Coull et al., 2013; Brodie et al., 2014). Brodie et al. (2014) identified time and resources as barriers to prescribing, as well as the act of prescription writing itself, which Bowden (2005) referred to as the 'chore' of writing prescriptions. This perception of prescribing as time-consuming was mirrored in the initiation and implementation of clinical management plans when using supplementary prescribing (Carey et al., 2007) and in the prescribing of controlled drugs (Maddox, 2011). In these instances, referring the patient to the GP was seen as a quicker method of ensuring the patient received the necessary medicines.

For some, the clinical setting added to the challenge of prescribing. Hall et al. (2006) found that the challenges of documenting the prescribing activity was exacerbated by the setting itself. Health visitors identified busy clinics as settings where prescribing was particularly difficult because of time constraints, with some nurse prescribers choosing not to reveal that they were able to prescribe. For others, the legal limitations of their prescribing rights and formulary limitations meant that they did not initiate prescribing, as they were concerned about providing a fragmented service (Davies, 2005). Although all the barriers identified so far have emerged from data collected from studies within the United Kingdom, Klein (2015) reported on Clinical Nurse Specialists in Oregon, with some similar findings. Although some of the barriers, such as insurance costs, clearly reflected the US system, others, such as role restrictions and time pressures, were consistent.

It appears that the barriers to prescribing are, at least, in part, attributable to a general lack of infrastructure to support prescribing (Scottish Government, 2009). Coull et al. (2013) identified fragmented implementation of prescribing policy that resulted in variations in implementation. Two of the initial barriers to prescribing were difficulty in getting a prescription pad and a lack of agreement regarding budgetary arrangements (Hall, 2006; Hall et al., 2006; Carey et al., 2007; Coull et al., 2013). The lack of preparation for prescribing was reflected in the need for some nurse prescribers to build relationships with GPs to gain agreement to prescribe across practices (Hall, 2006; Carey et al., 2014) and some nurses experienced explicit objection to their prescribing (Hall, 2006; Hall et al., 2006). Interestingly, even though they were able to identify a number of

Primary Health Care Research \& Development 2018; 19: 7-22 benefits to the patient and to their role, a number of studies made reference to the nurse prescriber's assertion that they undertook the role of prescriber without 'reward' through grade or pay (Davies, 2005; Scottish Government, 2009; Downer and Shepherd, 2010; Cousins and Donnell, 2012; Darvishpour et al., 2014). This desire for recognition by many nurse prescribers of the additional work and responsibility that nurse prescribing brings, may be associated with their perception of the nursing role itself.

\section{Role preservation}

A concept represented in a third of the studies reviewed (14 qualitative, three mixed-methods and a metasynthesis) was that of 'role', with a desire to preserve the nursing aspect of this. Prescribing was seen as an essential component of the nurses' role, particularly for specialist nurses (Bowskill, 2009; Carey et al., 2014). The nurse prescribers in Maddox's (2011) study stated they would not prescribe if they did not feel that it was within their role, yet the perception of how prescribing was accommodated within a nursing role varied. Bradley and Nolan's (2007) study produced some rich data in relation to this issue, with some nurses feeling it legitimised their role by enabling them to 'integrate caring and curing', a perspective mirrored by Mahoney and Ladd (2010) who identified that nurses felt they were 'more than a prescriber'; however, some nurses did make a distinction between prescribing and nursing, using terms such as 'spending more time on prescribing than nursing' and 'wearing lots of different hats', demonstrating that some nurses did not see it as integral to their nursing role (Bradley and Nolan, 2007). What appears to emerge from many studies is that there is a desire to differentiate the role of the nurse prescriber from that of the doctor.

'Medicalisation' of the nursing role was identified as a concern by nurses in a number of studies (Bradley and Nolan, 2007; Scottish Government, 2009; Coull et al., 2013), with a clear resistance to the suggestion that prescribing leads to medicalisation (Davies, 2005; Bowskill, 2009; Stenner et al., 2010a; 2010b). Brodie et al. (2014) identified that in view of prescribing moving from an activity once exclusive to doctors, there had to be a necessary shift in power to enable this, reflecting what Carey 
et al. (2010) termed 'shared territory'. Indeed, prescribing was found to bring with it more job control and enhanced status (Cousins and Donnell, 2012), suggesting a strengthening of the professional role of nurses through increased status and trustworthiness (Wilhelmsson and Foldevi, 2003), increased recognition and respect (Scottish Government, 2009; Coull et al., 2013; Darvishpour et al., 2014) and an 'increased credibility and confidence to recommend medicines' (Stenner and Courtenay, 2008b).

\section{Power-shifts and inter-professional relationships}

Of the papers reviewed, 17 that included five mixed-method papers, a metasynthesis and 11 qualitative studies presented data that were representative of the theme of 'power-shifts and inter-professional relationships', identifying a need for adjustments in professional relationships in order to enable effective implementation of prescribing. Bowden (2005) found that nurse prescribers experienced inappropriate expectations from other health-care professionals, indicating a lack of understanding of the professional and legal boundaries of the role. Further, some prescribers experienced resistance, which some perceived as professional rivalry (Bowskill, 2009). Although nurse prescribers felt support from nursing colleagues was desirable (though not essential) (Bowskill, 2009) when prescribing in more complex situations in which clinical decisions were perceived to be of a higher risk, a team approach to prescribing was seen as a necessity (Lewis-Evans and Jester, 2004; Stenner and Courtenay, 2008a; Carey et al., 2010; Stenner et al., 2010a; 2010b).

Indeed, nurse prescribing has provided an opportunity to develop relationships with other health professionals (DH, 2011; Coull et al., 2013; Darvishpour et al., 2014), with the nurse-pharmacist relationship most frequently cited (Bowden, 2005; Hall, 2006; Bradley et al., 2007; Stenner and Courtenay, 2008a). Pharmacists are perceived by NMPs to have a key role in safety (Bradley et al., 2007) and are a key source of support (Klein, 2015). Similarly, the nurse prescriber-doctor relationship was seen as important in supporting prescribing (Bradley et al., 2007; Northern Ireland Practice and Education Council for nursing and midwifery (NIPEC), 2007; Bowskill, 2009; Stenner et al., 2010a; 2010b). Some nurse prescribers encountered a lack of understanding by doctors of the boundaries of their role and their awareness of budgetary issues (Wilhelmsson and Foldevi, 2003; Carey et al., 2010), sometimes to the extent that they would not support nurse prescribing (NIPEC, 2007). In addition, some nurse prescribers perceived that doctors considered the prescribing rights of nurses as a threat to their own profession (Bradley et al., 2007) and that prescribing should be exclusive to their profession (Wilhelmsson and Foldevi, 2003), to the extent of employing financial or managerial control on nurse prescribing without having any authority to do so (Fisher, 2010); however, others had a more positive experience, with doctors trusting nurse prescribers to set their own boundaries (Carey et al., 2007; Bowskill, 2009; Mahoney and Ladd, 2010). Prescribing was felt to have given nurses increased confidence to debate with doctors (Bradley et al., 2007) and provided more opportunity for co-operation between them (Wilhelmsson and Foldevi, 2003). Indeed, it was recognised that new boundaries of practice had to be agreed in order to work collaboratively and to enable nurses to define their prescribing role (Bowskill, 2009).

\section{Culture of prescribing}

It appears that for nurse prescribing to flourish, it needs to be recognised, understood and be integral to the organisation's processes, demonstrating a culture supportive of prescribing; a theme that emerged in six of the included qualitative studies as well as in two of the mixed-method studies and a literature review. A clear policy on NMP, access to CPD, formal support mechanisms and a learning environment that encourages knowledge sharing across professions and the opportunity to influence policy were perceived as key factors indicative of a supportive organisation (Stenner and Courtenay, 2008a). The ease with which nurse prescribing is implemented appears to be influenced by how prepared the organisation was in relation to CPD, structures and processes (Jones et al., 2007; Stenner and Courtenay, 2008a; Stenner et al., 2010b). Stenner et al. (2010b) also made reference to support structures and culture, identifying that a range of CPD mechanisms were needed to achieve structures and cultures supportive of nurse prescribing. Where good 
inter-professional working relationships existed, this helped to promote a supportive culture for NMP and the support of the doctor was seen as key to this; however, the large-scale study by $\mathrm{DH}$ (2011) found that only half of the Trusts had a strategy for the development of NMP and that it was largely driven by the practitioner to enhance existing services rather than to enable service redesign, suggesting that its benefits are not yet fully appreciated in some areas. Indeed, even when the benefits are recognised by patients, colleagues and stakeholders alike, there is still evidence of fragmentation in policy and implementation (Coull et al., 2013).

\section{Limitations}

One of the key objectives of undertaking this metasynthesis was to provide context for the author's subsequent research exploring the lived experience of the nurse prescriber in primary care, utilising a phenomenological approach. As such, a broad approach was applied to the literature search to capture the wide range of possible influences on nurse prescribing. In doing so, mixedmethod studies were included; however, because of the limited participant quotes provided in some of these papers, it was not always possible to clearly determine whether the themes identified by the authors originated from qualitative data, quantitative data or a combination of both.

\section{Summary and implications}

This metasynthesis was undertaken in order to gain an insight into the factors that might influence the lived experience of the prescriber. Only a limited number of studies used a research methodology appropriate for exploring the lived experience to the depth associated with a phenomenological approach; thus, the decision to include other qualitative studies was important in order to provide sufficient context for further research. Unsurprisingly, in view of the numerous settings and specialities in which nurse prescribers practice in primary care, a wide range of themes emerged. This was reflected in the metasynthesis of literature reviews and systematic reviews in nurse prescribing undertaken by Darvishpour et al. (2014), who identified eight themes from the 11 papers included.

Primary Health Care Research \& Development 2018; 19: 7-22
As the health service remains under increasing financial pressures, staff are increasingly required to identify positive outcomes from the interventions they undertake. As such, it is to be expected that many of the studies in nurse prescribing identify benefits to the patient and to the service, with the recent systematic review undertaken by Weeks et al. (2016) reflecting this; however, of particular interest in this metasynthesis is the understanding that nurse prescribers not only appear to express a need to benefit the patient in order to validate their prescribing but also that they have an inherent need to provide patientcentred care, although this is not directly explored in the studies.

A number of themes emerged from the review of these studies, which influenced the scope of the nurse prescriber's prescribing activity, and many of these were reflective of the professional and ethical frameworks associated with nursing. These themes include 'professional accountability and boundary setting' and 'the need for knowledge'. Perhaps, not surprisingly, safety was also a consideration but it appeared to present itself as a 'safety consciousness' that spanned the breadth of the prescribing process, rather than simply as an awareness of safety issues. The source of this safety consciousness was not directly explored in any of the studies.

Relationships and their importance to prescribing was identified, with the nurse prescriberdoctor and the nurse prescriber-pharmacist relationships having some prominence. The challenges of taking on a role that was once exclusive to doctors, appear to have resulted in some internal conflicts regarding identity in some practitioners, leading to activities to support 'role preservation'. A number of studies provided a picture of what the issues relating to role are but the impact of these on the experience of the nurse prescriber was unclear. The context in which these challenges exist and how organisations and teams can support prescribing was also explored in some of the studies. It appears that the research to date is suggestive of the need for an environment in which prescribing is embraced in order for it to be effective; however, this has to be supported by a range of other factors such as policy, recognition and knowledge sharing. In other words, it would appear that a culture shift is needed, and although the literature often addresses the issues 
individually, the concept of culture warrants further consideration and it is evident that more needs to be discovered about nurse prescribing in order to effectively support it.

The systematic review of quantitative studies undertaken by Gielen et al. (2014), which made comparisons between physician and nurse prescribing, provides a useful perspective from which to consider this metasynthesis. Although Gielen et al.'s (2014) quantitative study maintained the need for further quantitative randomised control trials, it did acknowledge that nurse prescribing is embedded in the context of other nursing activity, which could impact the findings. As such, this metasynthesis of qualitative studies offers an insight into the issues and influences which contribute to that context.

\section{Acknowledgement}

The authors express thanks to the supervisory team for their support and contributions to the quality assessment process.

\section{Financial Support}

This metasynthesis was undertaken as part of MPhil/PhD studies that were partially funded by the Continuing Professional Development monies from the Faculty of Health and Wellbeing, University of Central Lancashire.

\section{References}

Bearman, M. and Dawson, P. 2013: Qualitative synthesis and systematic review in health professions education. Medical Education 47, 252-60.

Bhanbhro, S., Drennan, V.M., Grant, R. and Harris, R. 2011: Assessing the contribution of prescribing in primary care by nurses and professionals allied to medicine: a systematic review of literature. BMC Health Services Research 11, 330.

Bissell, P., Cooper, R., Guillaume, L., Anderson, C., Avery, A., Hutchinson, A., James, V., Lymn, J., Marsden, E., Murphy, E., Ratcliffe, J., Ward, P. and Woolsey, I. 2008. An evaluation of supplementary prescribing in nursing and pharmacy. London: TSO.

Bowden, L. 2005: The impact of nurse prescribing on the role of the district nurse. Nurse Prescribing 3, 79-86.

Bowskill, D. 2009: The integration of nurse prescribing: case studies in primary and secondary care. EthoS. Retrieved 20 April 2015 from http://ethos.bl.uk/SearchResults.do.

Bowskill, D., Timmons, S. and James, V. 2013: How do nurse prescribers integrate prescribing in practice: case studies in primary and secondary care. Journal of Clinical Nursing 22, 2077-86.

Bradley, E., Hynam, B. and Nolan, P. 2007: Nurse prescribing: reflections on safety in practice. Social Science and Medicine 65, 599-609.

Bradley, E. and Nolan, P. 2007: Impact of nurse prescribing: a qualitative study. Journal of Advanced Nursing 59, 120-28.

Brodie, L., Donaldson, J. and Watt, S. 2014: Non-medical prescribers and benzodiazepines: a qualitative study. Nurse Prescribing 12, 353-59.

Carey, N., Courtenay, M. and Burke, J. 2007: Supplementary nurse prescribing for patients with skin conditions: a national questionnaire survey. Journal of Clinical Nursing 16, 1230-237.

Carey, N., Stenner, K. and Courtenay, M. 2010: How nurse prescribing is being used in diabetes services: views of nurses and team members. Journal of Nursing and Healthcare of Chronic Illnesses 2, 13-21.

Carey, N., Stenner, K. and Courtenay, M. 2014: An exploration of how nurse prescribing is being used for patients with respiratory conditions across the East of England. BMC Health Services Research 14, 27.

Coull, A., Murray, I., Turner-Halliday, F. and Watterson, A. 2013: The expansion of nurse prescribing in Scotland: an evaluation. British Journal of Community Nursing 18, 234-42.

Courtenay, M., Carey, N. and Burke, J. 2006: Independent extended and supplementary nurse prescribing practice in the UK: a national questionnaire survey. International Journal of Nursing Studies 44, 1093-101.

Cousins, R. and Donnell, C. 2012: Nurse prescribing in general practice: a qualitative study of job satisfaction and workrelated stress. Family Practice 29, 223-27.

Darvishpour, A., Joolaee, S. and Cheraghi, M.A. 2014: A metasynthesis study of literature review and systematic review published in nurse prescribing. Medical Journal of the Islamic Republic of Iran 28, 77.

Davies, J. 2005: Health visitors' perceptions of nurse prescribing: a qualitative field work study. Nurse Prescribing 3, $168-72$.

Department of Health (DH). 2011: Evaluation of nurse and pharmacist independent prescribing in England. London: TSO.

Downe, S. 2008: Metasynthesis: a guide to knitting smoke. Evidence Based Midwifery 6, 4-8.

Downe, S., Simpson, L. and Steen, M. 2009: Template for metasynthesis of qualitative research studies. Retrieved 4 March 2015 from sdowne@uclan.ac.uk

Downer, F. and Shepherd, C.M. 2010: District nurses prescribing as nurse independent prescribers. British Journal of Community Nursing 15, 348-52.

Earle, E.A., Taylor, J., Peet, M. and Grant, G. 2011: Nurse prescribing in specialist mental health (Part 1): the views and experiences of practising and non-practising nurse prescribers and service users. Journal of Psychiatric and Mental Health 18, 189-97.

Primary Health Care Research \& Development 2018; 19: 7-22 
Erwin, E.J., Brotherson, M.J. and Summers, J.A. 2011: Understanding qualitative metasynthesis: issues and opportunities in early childhood intervention research. Journal of Early Intervention 33, 186-200.

Fisher, R.S.N.S. 2010: Nurse prescribing: a vehicle for improved collaboration, or a stumbling block to inter-professional working? International Journal of Nursing Practice 16, 579-85.

Gielen, S.C., Dekker, J., Francke, A.L., Mistiaen, P. and Kroezen, M. 2014: The effects of nurse prescribing: a systematic review. International Journal of Nursing Studies 51, 1048-61.

Hacking, S. and Taylor, J. 2010: An evaluation of the scope and practice of non-medical prescribing in the North West. Retrieved 18 February 2016 from https://www.hecooperative.co.uk/sites/default/files/attachments/pages/hacking.pdf

Hall, J. 2006: Influences on community nurse prescribing. EthoS. Retrieved 22 April 2015 from http://ethos.bl.uk/ SearchResults.do

Hall, J., Cantrill, J. and Noyce, P. 2003: Influences on community nurse prescribing. Nurse Prescribing 1, 127-32.

Hall, J., Cantrill, J. and Noyce, P. 2006: Why don't trained community nurse prescribers prescribe? Journal of Clinical Nursing 15, 403-12.

Hall, J., Noyce, P. and Cantrill, J. 2008: Why do district nurse prescribers alter their prescribing patterns? British Journal of Community Nursing 13, 507-13.

Harris, J. and Taylor, J. 2004. Research literature review on prescribing. Edinburgh: The Scottish Government.

Health Education North West. 2015: NMP economic evaluation. Retrieved 11 October 2016 from https://www.hee.nhs.uk/ sites/default/files/documents/Agenda \%20Item \%207\%20-\% 20 i5 \%20Health\%20-\%20NMP\%20Economic\%20Evaluation. pdf

Jones, M., Bennett, J., Lucas, B., Miller, D. and Gray, R. 2007: Mental health nurse supplementary prescribing: experiences of mental health nurses, psychiatrists and patients. Journal of Advanced Nursing 59, 488-96.

Klein, T. 2015: Clinical nurse specialist prescriber characteristics and challenges in Oregon. Clinical Nurse Specialist CNS 29, 156-65.

Kroezen, M., Francke, A.L., Groenewegen, P.P. and van Dijk, L. 2012: Nurse prescribing of medicines in Western European and Anglo-Saxon countries: a survey on forces, conditions and jurisdictional control. International Journal of Nursing Studies 49, 1002-12.

Latter, S., Smith, A., Blenkinsopp, A., Nicholls, P., Little, P. and Chapman, S. 2012: Are nurse and pharmacist independent prescribers making clinically appropriate prescribing decisions? An analysis of consultations. Journal of Health Services Research and Policy 17, 149-56.

Lewis-Evans, A. and Jester, R. 2004: Nurse prescribers' experiences of prescribing. Journal of Clinical Nursing 13, 796-805.
Luker, K. A. and McHugh, G. A. 2002: Nurse prescribing from the community nurse's perspective. International Journal of Pharmacy Practice 10, 273-80.

Maddox, C. 2011: Influences on non-medical prescribing: nurse and pharmacist prescribers in primary and community care. EThoS. Retrieved 22 April 2015 from http://ethos.bl.uk/ SearchResults.do

Mahoney, D.F. and Ladd, E. 2010: More than a prescriber: gerontological nurse practitioners' perspectives on prescribing and pharmaceutical marketing. Geriatric Nursing 31, 17-27.

Nolan, P. and Bradley, E. 2007: The role of the nurse prescriber: the views of mental health and non-mental health nurses. Journal of Psychiatric and Mental Health Nursing $14,258-66$.

Northern Ireland Practice and Education Council for nursing and midwifery (NIPEC). 2007: Review of the implementation of the nurse prescriber role. Retrieved 20 April 2015 from http://www.nipec.hscni.net/Image/SitePDFS/Nurse PrescribingFinalRpt.pdf

Royal College of Nursing. 2012: RCN factsheet nurse prescribing in the $U K$. London: RCN.

Scottish Government. 2009: An evaluation of nurse prescribing in Scotland. Edinburgh: TSG.

Stenner, K., Carey, N. and Courtenay, M. 2010a: How nurse prescribing influences the role of nursing. Nurse Prescribing 8, 29-34

Stenner, K., Carey, N. and Courtenay, M. 2010b: Implementing nurse prescribing: a case study in diabetes. Journal of Advanced Nursing 66, 522-31.

Stenner, K. and Courtenay, M. 2008a: The role of inter-professional relationships and support for nurse prescribing in acute and chronic pain. Journal of Advanced Nursing 63, 276-83.

Stenner, K. and Courtenay, M. 2008b: Benefits of nurse prescribing for patients in pain: nurses' views. Journal of Advanced Nursing 63, 27-35.

Walsh, D. and Downe, S. 2005: Meta-synthesis method for qualitative research: a literature review. Journal of Advanced Nursing 50, 204-11.

Walsh, D. and Downe, S. 2006: Appraising the quality of qualitative research. Midwifery 22, 108-19.

Watterson, A., Turner, F., Coull, A., Murray, I. and Boreham, N. 2009. An evaluation of the expansion of nurse prescribing in Scotland. Edinburgh: The Scottish Government Social Research Department.

Weeks, G., George, J., Maclure, K. and Stewart, D. 2016: Non-medical prescribing versus medical prescribing for acute and chronic disease management in primary and secondary care. Cochrane Database of Systematic Reviews 11, CD011227.

Wilhelmsson, S. and Foldevi, M. 2003: Exploring views on Swedish district nurses' prescribing - a focus group study in primary health care. Journal of Clinical Nursing 12, 643-50. 